Esternalizzazione e centralizzazione dei processi di approvvigionamento: risultati e criticità dell'esperienza degli Estav nella sanità toscana

Cinzia Panero, Carmen Calabrese, Cristina Campanale, Milena Vainieri, Sabina Nuti*

\footnotetext{
* Cinzia Panero assegnista di ricerca, Cristina Campanale e Milena Vainieri dottorande, Carmen Calabrese borsista, Laboratorio Management e Sanità della Scuola Superiore Sant'Anna di Pisa.

Sabina Nuti Professore Associato di Economia e Gestione delle Imprese e Direttore Laboratorio Management e Sanità, Scuola Superiore Sant'Anna di Pisa.

Per la corrispondenza: Cinzia Panero, Laboratorio Management e Sanità, via San Francesco 18, 56127 Pisa. Indirizzo email: c.panero@sssup.it

Benché frutto di un intenso lavoro comune tra gli autori, i par. 1 e 7 sono attribuibili a Sabina Nuti, il par. 2 a Cinzia Panero, i par. 3 e 4 a Milena Vainieri; il par. 5 è stato sviluppato da Sabina Nuti, Cinzia Panero e Milena Vainieri, mentre al par. 6 hanno collaborato Carmen Calabrese, Cristina Campanale e Cinzia Panero.

Ringraziamenti

Si ringrazia il management delle aziende sanitarie toscane e degli Estav che hanno collaborato al progetto di ricerca ed in particolare le dott.sse Cecilia Chiarugi e Carla Donati componenti dello staff direttivo della Regione Toscana, il Prof. Niccolò Persiani dell'Università di Firenze per gli spunti di riflessione forniti, nonché la dott.ssa Anja Gepponi dell'Università di Siena per la fattiva collaborazione.
} 


\title{
Esternalizzazione e centralizzazione dei processi di approvvigionamento: risultati e criticità dell'esperienza degli Estav nella sanità toscana
}

\begin{abstract}
Le opportunità ed i vantaggi derivanti dall'esternalizzazione e dalla centralizzazione dei processi aziendali, evidenziati in letteratura nel contesto privato, sono riscontrabili anche nel contesto sanitario pubblico? L'articolo si propone di compiere una prima valutazione dei risultati raggiunti in Toscana con l'introduzione degli ESTAV, la cui istituzione è avvenuta per razionalizzare, a livello sovra-aziendale, le funzioni tecnico-amministrative ed in particolare gli approvvigionamenti. Valutando i risultati raggiunti in termini di efficienza e di efficacia, viene evidenziato come tale esperienza presenti, pur con ampi spazi di miglioramento, risultati indubbiamente positivi e assimilabili a quelli presenti nel contesto privato.
\end{abstract}

Parole chiave: approvvigionamento - centralizzazione - esternalizzazione - sanità servizi pubblici - efficienza

Centralization and outsourcing of purchasing processes: results and criticalities of Estav in the Tuscany health care system

Considering the opportunities, pointed out by the literature, that the process outsourcing and centralization can offer in the private sector, this paper aims to evaluate the Tuscany experience in the public health system. Through the institution of ESTAVs, the Tuscany health system has introduced a co-operative level among the LHAs to achieve the rationalization of purchasing and technical functions.

The paper, after the introduction of the adopted methodology, illustrates the results achieved by ESTAVs in terms of efficiency and effectiveness, with particular reference to the cost savings and the impact on market competition. The results confirm a positive evaluation, even if there is space for improvements.

Keywords: outsourcing - centralization - purchasing - health system - public services - efficiency

\section{Introduzione}

Da tempo le imprese sono consapevoli della rilevanza strategica degli approvvigionamenti (Spekman et al., 1994; Hakansson e Gadde, 2002), sia perché mediamente spendono dal $50 \%$ all' $85 \%$ del loro fatturato in acquisti (Momme e Hvolby, 2002), sia perchè questi ultimi costituiscono un elemento chiave nel processo di creazione di valore per il cliente (Tunisini, 2003). Tale rilevanza, e la presenza di fattori che accentuano l'esigenza di interazione nella supply chain (Cozzi e Ferrero 
2004), hanno quindi indotto a sviluppare specifiche modalità gestionali con cui rapportarsi ai fornitori, differenziate in funzione dell'importanza del prodotto/servizio oggetto della fornitura e del coinvolgimento nella relazione con il fornitore (Kralijc, 1983; Anderson e Narus, 2004).

Anche in sanità si sta sempre più diffondendo la consapevolezza circa la rilevanza degli acquisti, in considerazione dell'impatto, a livello di efficacia e di economicità, che hanno sulla qualità dei processi produttivi.

La funzione acquisti, a lungo considerata di secondo piano, separata dalle altre e finalizzata alla mera gestione operativa dei flussi di ordini e consegne con i fornitori, si sta sempre più connotando come funzione strategica che supporta l'impresa nel perseguire un vantaggio competitivo (Gadde, Hakansson, 1994; Hines, 1996) e, nelle organizzazioni dotate di differente finalità caratteristica, quali le Aziende Sanitarie, nel conseguire una maggiore efficienza ed efficacia.

Negli ultimi anni la crescente attenzione dedicata in sanità all'impiego delle risorse disponibili e la ricerca di una maggiore efficacia nello svolgimento delle attività hanno pertanto alimentato molteplici tentativi di sperimentazioni ed innovazioni organizzative (Turchetti, 2005), soprattutto con riferimento alla gestione delle funzioni amministrative e degli approvvigionamenti.

I processi di acquisto, con il loro impatto sui bilanci delle Aziende sanitarie, richiedono infatti alla direzione aziendale uno sforzo continuo al fine di ricercare soluzioni e modelli capaci di garantire una maggiore efficienza sia nell'acquisto sia nella gestione della logistica dei beni e servizi (Brusoni e Marsilio, 2007). Rientrano in questa logica alcune iniziative intraprese negli ultimi anni in sanità, riguardanti l'esternalizzazione (outsourcing) e la centralizzazione, anche attraverso aggregazioni interaziendali, di alcune funzioni. Con queste iniziative anche in sanità si è verificato il passaggio dagli assetti istituzionali di tipo gerarchico a quelli a rete (Del Vecchio e Rossi, 2004; Del Vecchio, 2005).

L'outsourcing consiste nell' affidamento di funzioni ed attività precedentemente svolte dall'impresa committente a soggetti esterni ed è caratterizzato dalla relazione di cooperazione (Quinn, 2000, Gregori, 2001, Scarsi, 2004) che si instaura tra soggetto esterno ed organizzazione outsoucee, in cui il fornitore si assume la responsabilità di una o più funzioni svolte precedentemente dallo stesso outsourcee. L'esternalizzazione è pertanto uno strumento che consente alle organizzazioni di ridimensionare lo spettro delle attività svolte per focalizzarsi sulle core competences (Scarsi, 2004), soprattutto se si considera la tendenza in atto, che vede il passaggio dall'outsourcing di singole attività, lontane dal core business (Macinati, 2007) a quello di intere funzioni ed aree, quali la gestione del personale e la logistica (Gregori, 2001). In ambito pubblico occorre peraltro distinguere il processo di esternalizzazione che prevede il trasferimento dei processi ad un altro soggetto, sempre appartenente all'amministrazione pubblica ${ }^{1}$, da quello che vede coinvolto

\footnotetext{
${ }^{1}$ Tale processo di esternalizzazione, pur conservando tutte le caratteristiche dell'outsourcing, viene definito con termine più preciso contracting in (Padovani, 2004).
} 
un'impresa privata ${ }^{2}$ : nel primo caso, infatti, tra i vincoli vanno ricomprese anche tutte le rigidità del sistema pubblico.

Tra le varie iniziative maturate in ambito pubblico è significativa l'esperienza sviluppata in Toscana con i Consorzi di Area Vasta (CAV), sostituiti poi dagli Enti per il Supporto Tecnico-Amministrativo di Area Vasta (ESTAV).

Assumendo come riferimento la letteratura in tema di esternalizzazione e centralizzazione dei processi aziendali, questo articolo si propone di compiere una prima valutazione dell'esperienza sviluppata con gli Estav in Toscana e quindi dei risultati raggiunti e raggiungibili, anche in un contesto pubblico, attraverso la scelta di esternalizzare e centralizzare alcune funzioni, in particolare gli approvvigionamenti.

La prima parte dell'articolo illustra, attraverso l'analisi della letteratura, le prospettive teoriche connesse alle pratiche di esternalizzazione e centralizzazione dei processi aziendali, evidenziandone i possibili vantaggi e le modalità gestionali idonee a garantirne il successo e limitarne i rischi. Segue quindi l'illustrazione delle caratteristiche degli Estav in Toscana, interpretandone, alla luce della letteratura analizzata, le finalità con cui sono stati istituiti e la missione loro affidata. La terza parte illustra la metodologia adottata per valutare i risultati raggiunti dagli Estav, che sono quindi presentati nella quarta parte e discussi, anche in relazione al quadro teorico iniziale, nella sezione conclusiva.

\section{L'outsourcing e la centralizzazione degli acquisti: prospettive teoriche}

\section{L'outsourcing e la centralizzazione}

Il tema relativo alle scelte di make or buy, e la conseguente definizione dei confini delle organizzazioni (Coase, 1937), è stato affrontato sotto più profili in letteratura, identificando, di volta in volta, nella minimizzazione dei costi di transazione (Williamson, 1975), nella necessità (o meno) di coordinare attività complementari o simili (Richardson, 1972) e di adattarsi alle trasformazioni dell'ambiente economico (anche attraverso l'individuazione di nuove modalità con cui organizzare gli scambi, Powell, 1987), nella focalizzazione sulle core competences (Prahalad e Hamel, 1990) gli elementi discriminanti a supporto di tale scelta.

Ma quali sono le ragioni sottostanti le decisioni di outsourcing?

La letteratura ha evidenziato le seguenti motivazioni principali:

- riduzione dei costi, come conseguenza dell'esternalizzazione di attività non core (Momme e Hvolby, 2002, Valentini, 1999), grazie al recupero di efficienza in attività a limitato valore aggiunto, anche mediante il ridimensionamento delle strutture di supporto (Gregori, 2001);

\footnotetext{
${ }^{2}$ Un esempio significativo è la decisione del Servizio Sanitario Inglese (NHS) di esternalizzare le funzioni logistiche, precedentemente svolte da una sua divisione (NHS Logistics) a DHL: il contratto provocò una discussione accesa circa l'effettuazione di una "parziale privatizzazione" del sistema sanitario nazionale (Schuller, Hubner, 2008).
} 
- maggiore flessibilità organizzativa (Gandolfi, 2000), in quanto la sostituzione della struttura gerarchica interna con una esterna, regolata tramite contratto, può consentire un rapido adeguamento alle variazioni di carico di lavoro (Valentini, 1999), modificando anche la struttura dei costi aziendali, di cui viene accresciuta la componente variabile rispetto a quella fissa (Gregori, 2001);

- focalizzazione sul core business: l'esternalizzazione delle attività marginali consente di liberare risorse che possono essere dedicate alle attività più rilevanti dell'organizzazione (Tayles e Drury, 2000), rafforzandone quindi l'azione nel proprio core business (Quinn e Hilmer, 1994, Venkatesan, 1992);

- accesso a investimenti, innovazioni, risorse e capacità specialistiche (Quinn e Hilmer, 1994). L'attività esternalizzata costituisce infatti il core business del fornitore che quindi tenderà ad investirvi le migliori risorse e tecnologie (Valentini, 1999; Gregori, 2001; Scarsi, 2004). In effetti la riduzione dei costi del servizio esternalizzato, individuata come prima motivazione dell'outsourcing, si connette all'ulteriore possibilità, per l'organizzazione outsourcee, di appropriarsi dei benefici derivanti dalle sinergie e dalle economie di scala che il fornitore può conseguire grazie alla sua maggiore dimensione e specializzazione ed allo sviluppo di conoscenze specifiche attraverso l'apprendimento. Sfumano quindi i confini tra le imprese e tra le risorse direttamente controllate e quelle non controllate, poiché mediante le relazioni molte unità esterne diventano risorse mobilitabili al servizio della strategia dell'organizzazione outsourcee;

- maggiore qualità dei servizi esternalizzati (Kakabadse e Kakabadse, 2001) e quindi maggiore competitività, come conseguenza dell'accesso ad una rete di specialisti (Quinn, 2000). In proposito è stato peraltro osservato che la reingegnerizzazione, tramite outsourcing, delle organizzazioni nel settore pubblico, ha portato a strutture più snelle, più efficienti, ma anche giudicate spesso fornitrici di servizi di qualità inferiore (Kakabadse e Kakabadse, 2001).

Anche la centralizzazione a livello sovraziendale, che negli ultimi anni ha accompagnato in sanità alcune iniziative di esternalizzazione nel settore degli approvvigionamenti (Brusoni et al., 2008), si pone come obiettivo principale il conseguimento di una forte riduzione della spesa per beni e servizi, attraverso:

- lo sfruttamento delle economie di scala derivanti dai maggiori volumi acquistati;

- $\quad$ il conseguimento di un potere di mercato superiore a quello precedentemente detenuto dalle singole Aziende (Meneguzzo et al., 2004, Momme e Hvolby, 2002), aspetto tanto più rilevante quanto più l'offerta risulta essere concentrata, come per esempio nel caso dei farmaci (Turchetti, 2005) ;

- la riduzione dei costi di transazione tra Aziende e fornitori, connessi alla raccolta e trasmissione di informazioni e di documenti amministrativi (Brusoni e Marsilio, 2007); 
- la riduzione del personale delle singole Aziende adibito alle funzioni oggetto di centralizzazione al loro esterno;

- la specializzazione del personale, attraverso la costituzione di unità adibite ad attività specifiche;

- la standardizzazione dei processi, che rende più efficiente il loro svolgimento.

La centralizzazione degli acquisti, in sanità, comporterebbe inoltre una serie di impatti di natura strategica (Brusoni et al., 2008), quali:

- la razionalizzazione del portafoglio fornitori e, conseguentemente, una gestione dei rapporti più efficiente e, grazie ai maggiori volumi, la possibilità di attrarre imprese precedentemente non interessate a rifornire le singole Aziende;

- la standardizzazione dei beni e dei servizi richiesti dalle Aziende, per potere procedere all'aggregazione della domanda, aspetto che richiede peraltro un notevole coinvolgimento delle Aziende e dei professionisti;

- le Aziende, inoltre, nello svolgimento delle loro attività, sarebbero supportate, grazie alla maggiore qualificazione del personale ed alla presenza di sistemi informativi condivisi, da una struttura amministrativa di supporto più efficiente ed efficace.

E' stato peraltro evidenziato che, se in fase di progettazione strategica le organizzazioni manifestano in genere un grande entusiasmo e grandi aspettative, si ha spesso poca cura nel gestire operativamente la relazione con il fornitore (Valentini, 1999), rispetto alla quale prevale spesso un atteggiamento di attesa passiva dei risultati.

L'outsourcing è in effetti complesso da gestire, a causa delle interdipendenze tra attività e funzioni da trasferire da un'organizzazione all'altra (Gadde e Jonsson, 2007) e della natura adversarial che, nei servizi pubblici (Kakabadse e Kakabadse, 2001), le relazioni cliente-fornitore rischiano di assumere.

In letteratura è stato in effetti sottolineato che l'outsourcing può comportare anche alcuni rischi, connessi alla corretta identificazione delle caratteristiche e dell'ampiezza delle attività da esternalizzare (a causa della difficoltà, da parte delle organizzazioni, di comprendere o definire in modo dettagliato le proprie esigenze, Gregori, 2001), alla gestione del rapporto con il fornitore, alle conseguenze derivanti dalla decisione di effettuare l'outsourcing (Gadde e Jonsson, 2007).

Più nel dettaglio, i problemi più rilevanti sono:

- problemi di gestione del rapporto con il fornitore, che può anche rivelarsi meno efficiente del previsto (Tales e Drury, 2000), o meno motivato rispetto agli obiettivi dell'organizzazione (Momme e Hvolby, 2002), con profonde conseguenze sulla qualità del servizio, sull'affidabilità nelle consegne e quindi sui tempi dei processi;

- riduzione dell'entità dei risparmi di spesa conseguiti, a causa della presenza di costi di coordinamento (Gandolfi, 2000);

- perdita di autonomia nei confronti del fornitore, a causa delle interdipendenze (tecnologiche, conoscitive, sociali, economiche), derivanti dalla condivisione di risorse e dagli adattamenti reciproci; 
- perdita di controllo sulle conoscenze, che comporterebbe una maggiore vulnerabilità dell'organizzazione (Scarsi 2004): il know-how e le competenze dell'organizzazione relativamente all'attività esternalizzata possono ridursi (Momme e Hvolby, 2002) e conseguentemente aumenta il rischio che la conoscenza diventi obsoleta e diminuisca la capacità di innovazione ed apprendimento (Gregori, 2001);

- perdita di motivazione del personale (Momme e Hvolby, 2002), a causa della percezione dell'outsourcing come di una minaccia alla propria attività ed alla scarsa fiducia nei fornitori, con conseguente possibilità che si verifichino forti resistenze interne. In particolare è stato rilevato che i manager del settore pubblico ritengono che l'outsourcing abbia un impatto negativo in misura maggiore dei manager del settore privato, anche per la minore discrezionalità nelle scelte organizzative ed il rischio di perdere la capacità di riconoscere i bisogni dell'organizzazione e della comunità (Kakabadse e Kakabadse, 2001).

Per ovviare ai rischi derivanti dall'esternalizzazione e centralizzazione delle attività e garantirne il successo, sono state proposte numerose indicazioni di natura gestionale. Se nel caso dell'outsourcing viene evidenziata la necessità di sviluppare una gestione accurata del rapporto con il fornitore, dall'individuazione delle attività da esternalizzare, alla definizione delle condizioni contrattuali, alla sua esecuzione e monitoraggio, nel caso della centralizzazione delle attività presso un soggetto terzo l'attenzione viene posta sulla necessità, da parte delle singole Aziende, di dotarsi di strutture e procedure adeguate (Brusoni et al., 2008).

La lettura congiunta delle indicazioni fornite in materia di outsourcing e centralizzazione può quindi individuare un possibile insieme di soluzioni atte a garantire il successo del processo di esternalizzazione e centralizzazione degli approvvigionamenti, oggetto di questo articolo.

In fase di avvio dell'iniziativa è opportuno:

- all'interno dell'Azienda outsourcee, identificare le attività da trasferire e definire i possibili effetti dell'esternalizzazione sull'organizzazione, sui flussi di lavoro, sui tempi e sul funzionamento della relazione con il centro che gestisce le attività per cui si è operato l'outsourcing (Gregori, 2001); inoltre le attività andranno sottoposte a revisione periodica, per verificarne la congruità rispetto alle mutate condizioni ambientali;

- $\quad$ tra le Aziende e l'ente centralizzatore:

- individuare meccanismi di governance dell'ente centralizzatore che coinvolgano le Aziende, la cui scarsa partecipazione viceversa può indebolirne la fiducia, aumentarne la percezione di scarsa utilità e di inefficienza, riducendo così le possibilità di successo;

- procedere alla standardizzazione dei beni acquistati e delle procedure. Essa richiede un'attenta programmazione aziendale, aspetto che può essere critico, soprattutto se le Aziende coinvolte sono numerose, i professionisti fanno valere la loro autonomia e specificità professionale e, per ovviare alle conseguenti difficoltà, 
vengono effettuati acquisti in economia, rischiando così di compromettere la centralizzazione.

Durante lo svolgimento dell'esternalizzazione/centralizzazione, il focus si sposta più sul coordinamento operativo tra le parti e lo sviluppo di adeguati strumenti di supporto all'interno delle Aziende. Più in particolare:

- diventa importante definire all'interno delle due imprese i soggetti (process owner) con il compito di mantenere i contatti tra le organizzazioni;

- occorre incentivare un flusso di comunicazione tra le parti, formale e non, allo scopo di monitorare l'evoluzione della relazione, di condividere conoscenze, di apprendere (Gregori, 2001; Scarsi, 2004) e quindi di sviluppare la fiducia, tanto più importante quanto più si considera che, comunque, non tutti gli aspetti del rapporto possono essere formalizzati ${ }^{3}$;

- formare i manager nella gestione delle relazioni con il fornitore, in particolare circa le modalità di integrazione e coordinamento delle due organizzazioni (procedure, infrastrutture e definizione dei sistemi di comunicazione) e lo sviluppo di commitment e motivazione nel personale;

- motivare il fornitore attraverso la partecipazione al rischio delle operazioni, stabilendo che la remunerazione sia connessa al raggiungimento di determinati obiettivi: in questo modo si evita anche il rischio di comportamenti opportunistici;

- mantenere il controllo sul know-how, per presiedere le competenze che consentano il monitoraggio sulle attività esternalizzate.

In tutte le fasi viene infatti sottolineata la necessità di effettuare la valutazione della performance dell'outsourcer, per determinare il successo o fallimento dell'iniziativa. L'impresa deve pertanto costruire con il fornitore un sistema di valutazione e di reporting dei risultati, che devono essere rilevati periodicamente ed elaborati in forma di indicatori per rendere efficace la valutazione della performance (Nuti, 2005) e rendere noti i risultati conseguiti. A tal fine è opportuno che vengano valutati il livello di soddisfazione dell'utilizzatore e gli effetti dell'iniziativa sulla gestione aziendale, evidenziandone benefici e problemi.

Queste indicazioni verranno confrontate con le soluzioni adottate per introdurre ed implementare in Toscana i Consorzi di Area Vasta (CAV), poi sostituiti dagli Enti per i Servizi Tecnico Amministrativi di Area Vasta (ESTAV), valutandone anche la capacità nel perseguire i vantaggi sopra indicati.

\section{Gli ESTAV in Toscana: le caratteristiche e l'evoluzione}

In Toscana il primo passo nella direzione della riorganizzazione del servizio sanitario in ottica interaziendale avviene con la legge di "Riordino delle norme per l'organizzazione del servizio sanitario regionale" (L.R. 22/2000) che individua le Aree Vaste come "la dimensione operativa a scala interaziendale ottimale" per

\footnotetext{
${ }^{3}$ Questo aspetto è ancora più rilevante nell'outsourcing strategico industriale, in cui il comaker non solo partecipa, ma può anche essere il principale attore dei processi innovativi più rilevanti.
} 
svolgere "atti di concertazione", anche con riferimento all'organizzazione ed alla gestione dei servizi.

E' stato osservato (Del Vecchio e Rossi, 2004; Turchetti, 2005) che con l'istituzione delle Aree Vaste si compie il passaggio da una fase segnata da una marcata competitività tra le Aziende ad una in cui prevale la logica della cooperazione interaziendale.

Questa logica infatti risponde meglio alla finalità di massimizzare il valore prodotto per il cittadino che, nel contesto sanitario, è in condizioni di asimmetria nei confronti del soggetto erogatore e quindi non in grado di essere il regolatore del mercato di scambio.

Attraverso la realizzazione del livello sovraziendale di Area Vasta, che include la gestione, programmazione e organizzazione in forma associata dei servizi, vengono inoltre perseguiti non solo la programmazione strategica del sistema, ma anche il miglioramento della sua efficienza.

Tale intento è stato confermato con la D.G.R. 144/2000 che istituiva, per ciascuna Area Vasta, un Consorzio di Area Vasta (CAV), avente natura di società consortile a responsabilità limitata, cui era affidata come missione la centralizzazione dei processi di acquisto, ma con la prospettiva di una futura estensione dell'attività ad altre funzioni di supporto.

L'obiettivo primario, sottostante l'istituzione dei Consorzi, era la razionalizzazione delle funzioni tecnico-amministrative (Cravedi, 2003) e la particolare attenzione data alla funzione acquisti era connessa al fatto che si presentava come il settore più maturo per realizzare una politica di razionalizzazione della spesa e di ottimizzazione delle risorse.

I CAV sono entrati in attività il $1^{\circ}$ gennaio 2003.

Lo svolgimento dell'attività istituzionalmente loro assegnata si è però rapidamente scontrata con l'opposizione delle Aziende che, temendo una forte riduzione del proprio ruolo e potere all'interno del sistema, come conseguenza dell'esternalizzazione degli acquisti, opponevano una forte resistenza al trasferimento di tali servizi. L'assenza di un assetto istituzionale forte ed autonomo rispetto alle aziende sanitarie (l'organo di governo era costituito dal Consiglio di Amministrazione, cui partecipavano i Direttori Generali delle Aziende sanitarie) limitava l'efficacia dell'azione dei CAV.

Per superare questi problemi, con la L.R. 40/2005, i CAV sono stati trasformati in Enti per i Servizi Tecnico Amministrativi di Area Vasta (ESTAV), ossia in enti del servizio sanitario regionale, dotati di natura giuridica pubblica ed una marcata autonomia organizzativa, gestionale, contabile e tecnica ${ }^{4}$.

Con tale provvedimento è stato confermato per ciascun Estav il bacino di riferimento, dato dalle Aziende sanitarie incluse nella rispettiva Area Vasta, ossia:

\footnotetext{
${ }^{4}$ In effetti la soluzione così individuata si distingue rispetto ad altre esperienze (Consip, Aree Vaste dell'Emilia-Romagna..) per una scelta "forte" sotto il profilo istituzionale. Sicuramente il fatto di aver superato la logica del Consorzio ha aumentato il potere nei confronti delle Aziende, ma sempre nell'ambito di un mercato interno: tutti i soggetti appartengono infatti al sistema pubblico regionale, con tutte le rigidità che lo caratterizzano.
} 
- Estav Centro Toscana: Azienda USL di Pistoia, Prato, Firenze, Empoli e le Aziende Ospedaliero-Universitarie Careggi e Meyer;

- Estav Nord Ovest: Azienda USL di Massa e Carrara, Lucca, Pisa, Livorno, Versilia e l'Azienda Ospedaliero-Universitaria di Pisa;

- Estav Sud-Est: Aziende USL di Siena, Arezzo e Grosseto e l'Azienda Ospedaliero-Universitaria di Siena.

Ogni Estav è dotato di un organo di governo autonomo, il Direttore Generale, le cui funzioni sono assimilabili a quelle dell'analoga figura presente nelle Aziende sanitarie. L'organo di raccordo con le Aziende, cui compete comunque la definizione dei fabbisogni, il monitoraggio dei consumi e la programmazione, è stato individuato nel Consiglio Direttivo composto dai Direttori Generali delle singole Aziende, con il compito, tra gli altri, di approvare il programma annuale e pluriennale delle attività dell'ente.

Sempre la L.R. 40/2005 ha inoltre identificato le aree di competenza degli Estav, tramite l'indicazione delle funzioni loro affidate:

- approvvigionamento beni e servizi;

- gestione dei magazzini e della logistica;

- gestione delle reti informative e delle tecnologie informatiche;

- gestione del patrimonio per le funzioni ottimizzabili in materia di manutenzioni, appalti e alienazioni;

- $\quad$ organizzazione e gestione delle attività di formazione del personale;

- gestione delle procedure concorsuali per il reclutamento del personale;

- gestione delle procedure per il pagamento del personale.

E' da osservare che con l'esternalizzazione di tali funzioni si accentua l'aspetto core delle Aziende sanitarie, ossia la fornitura di servizi alla persona (Dominijanni e Nante, 2007), anche se, almeno inizialmente, il trasferimento delle funzioni è avvenuto solo con riferimento al processo d'acquisto.

Dal 17 ottobre 2005 (D.G.R. 1021/2005) gli Estav sono subentrati ai Cav.

Il trasferimento delle funzioni indicate dalla L.R. 40/2005 è ancora in corso: dopo la DG.R. 617/2006 che stabiliva il trasferimento della gestione ordini, della logistica e dei magazzini, la sua implementazione è stata quasi completata con la DG.R. $317 / 2007$ che prevede il trasferimento della gestione delle reti informative e delle tecnologie informatiche; della gestione del patrimonio per le funzioni ottimizzabili in materia di manutenzioni, appalti e alienazioni, delle procedure concorsuali per il reclutamento del personale e del pagamento del personale.

\section{Le premesse all'analisi dei risultati}

Avendo come riferimento la letteratura riguardante le decisioni di esternalizzazione e centralizzazione, quali vantaggi si sono voluti perseguire attraverso l'introduzione dei CAV e degli Estav?

Un primo tipo di vantaggio è indubbiamente di natura economica.

In particolare è stato evidenziato come l'outsourcing e la contemporanea centralizzazione degli acquisti consentano di conseguire economie di scala, con la 
possibilità di realizzare processi più efficienti sia per il fornitore sia per l'acquirente (Del Vecchio e Rossi, 2004; Dominijanni e Nante, 2007; Meneguzzo et al., 2004); tali economie possono essere ricollegate anche ai maggiori volumi acquisiti, e al conseguente maggior potere di mercato (Del Vecchio e Rossi, 2004).

Le possibilità di riduzione dei costi sarebbero inoltre favorite dalla specializzazione degli Estav che, sia a livello strutturale (con la costituzione delle sezioni consortili focalizzate su specifiche tipologie di beni e servizi), sia a livello di personale, dovrebbe consentire di approfondire la conoscenza dello specifico settore e delle sue dinamiche e quindi favorire lo sfruttamento del maggiore peso negoziale (Del Vecchio e Rossi, 2004; Meneguzzo et al., 2004; Del Vecchio 2005).

Più incerta appare il contenimento della spesa tramite la diminuzione dei costi di transazione, in quanto la demoltiplicazione dei contatti e della trasmissione di informazioni avviene più nell'interfaccia tra Estav e fornitori che non tra Estav e Aziende.

Un secondo aspetto che si può osservare è che con l'Estav si realizza una vera e propria organizzazione a rete, in cui avvengono processi di condivisione delle informazioni (Del Vecchio 2005). A tal proposito viene sottolineata l'importanza della presenza dei professionisti delle Aziende sanitarie nelle commissioni tecniche che predispongono $\mathrm{i}$ capitolati e che valutano le offerte dei fornitori: per loro si aprirebbe l'opportunità di accedere a conoscenze più ampie e, anche attraverso il confronto delle diverse prassi cliniche, di ridurre l'autoreferenzialità, in un contesto che attenua le possibilità di comportamenti opportunistici basati sul potere detenuto dal singolo all'interno della propria Azienda. La centralizzazione, infatti, consentirebbe di realizzare un maggior controllo sulla trasparenza delle procedure (Brusoni e Marsilio, 2007).

E' peraltro da osservare che tali risultati sono raggiungibili se e nella misura in cui le Aziende modificano gli assetti organizzativi, attraverso la standardizzazione dei processi, effettuando in maniera consona la programmazione, definendo in tempi utili le necessità. In particolare la soluzione adottata richiede che le Aziende abbiano sviluppato adeguati sistemi di budget e programmazione degli acquisti, così da governare i consumi (Del Vecchio e Rossi, 2004; Del Vecchio 2005). L'Estav si sostituisce infatti all'Azienda durante la gara, può stimolare una programmazione più sistematica, ma non svolgerla per conto della singola Azienda (Brusoni e Marsilio, 2007).

L'outsourcing, che di fatto si è attuato con l'introduzione degli Estav, comporta inoltre problemi circa la riallocazione delle attività, del personale e delle responsabilità precedentemente all'interno di un'unica Azienda. Comporta anche la necessità di aggregare le informazioni (che devono perciò essere le più omogenee possibile e supportate da un adeguato sistema informativo) ed i processi d'acquisto delle diverse Aziende che, per ragioni storiche, sono necessariamente differenti tra loro.

In tal senso un ulteriore problema che può emergere è che gare di maggiore ampiezza sono anche più complesse e quindi, rischiano di richiedere tempi maggiori (Brusoni e Marsilio, 2007), aspetto sul quale si possono appuntare le resistenze dei professionisti. Questi infatti, vista la profonda connessione esistente tra pratica medica e tecnologia e 
l'impatto che il processo di esternalizzazione ha su di essa, possono considerare minacciata la propria professionalità e, soprattutto, il proprio potere all'interno delle Aziende.

\section{La metodologia di ricerca adottata}

La ricerca si è proposta di valutare se la scelta di esternalizzare e centralizzare alcune funzioni presso gli Estav ha consentito di raggiungere gli obiettivi sopra individuati e quale è stato l'impatto sul sistema regionale. Per compiere questa valutazione è stata svolta un'analisi sistematica dell'attività dei tre Estav, attraverso la lettura di tutti gli atti aventi impatto economico (delibere di acquisto, rinnovo, estensioni...) effettuati dai tre Enti, per un arco temporale di tre anni.

Il primo progetto pilota, condotto nel 2006 ed avente come oggetto l'attività del 2005 , infatti, aveva evidenziato che una delle maggiori criticità, che rendeva problematico il confronto della performance tra $\mathrm{i}$ tre Estav, era la differente metodologia di registrazione degli atti.

Sulla base dell'analisi di tutti gli atti aventi impatto economico è stato quindi costruito un database, con caratteristiche omogenee, per ciascun Estav, contenente tutti gli aspetti salienti degli atti di aggiudicazione, per gli anni 2006 e 2007. Tale approccio ha reso possibile procedere ad una corretta comparazione dei risultati nello spazio (tra Estav) e nel tempo (dal 2005 al 2007).

Le dimensioni valutative selezionate si riferiscono in primo luogo al raggiungimento della missione affidata agli Estav, ossia alla loro capacità di conseguire un risparmio di risorse rispetto alla situazione precedente.

Un secondo aspetto sottoposto ad indagine è stato il livello di efficienza nello svolgimento delle attività, individuabile tramite:

- le tipologie di atto impiegate (per evidenziare quanta parte dei volumi acquistati derivino da aggiudicazioni derivanti da attività dell'Estav anzichè da proroghe di contratti preesistenti delle Aziende);

- la dimensione economica degli atti (con cui si evidenzia l'eventuale presenza di atti "sottodimensionati");

- l'efficienza nell'impiego del personale.

Infine sono stati analizzati aspetti segnaletici dell'efficacia degli Estav, ossia:

- la durata delle gare;

- il numero di fornitori invitati per gara (come proxi dell'apertura e competitività del mercato e della razionalizzazione del portafoglio fornitori).

Più nel dettaglio, le metodologie adottate per ciascuna rilevazione sono state:

Tab. 1 - La metodologia adottata

\begin{tabular}{lrl}
\hline Oggetto di analisi & Metodologia \\
\hline $\begin{array}{l}\text { Numero atti e loro } \\
\text { composizione }\end{array}$ & $\begin{array}{l}\text { Lettura degli atti aventi impatto economico di tutti e tre gli } \\
\text { Estav (anni 2005 -2007) e loro aggregazione per categorie } \\
\text { omogenee (anni 2006 -2007) }\end{array}$ \\
Volumi aggiudicati & Lettura degli atti aventi impatto economico di tutti e tre gli
\end{tabular}


Personale

Risparmio conseguito

Durata gare

N. fornitori invitati per gara
Estav, costruzione di un database omogeneo per anno di competenza (anni 2005-2007)

Raccolta dati sul personale dell'Estav (anni 2005-2006)

Nel progetto pilota relativo all'anno 2005: selezione di un campione di atti eterogenei per tipologia di bene aggiudicato e metodo di calcolo del risparmio, coinvolgenti una pluralità di Aziende e da cui derivasse una percentuale elevata del risparmio dichiarato.

Nella valutazione relativa all'anno 2007: selezione di tutti gli atti relativi a nuove aggiudicazioni in cui fossero presenti tutte le Aziende.

In ambedue i casi la valutazione del risparmio sugli acquisti è avvenuta valorizzando le quantità indicate negli atti al prezzo di aggiudicazione dell'Estav e confrontando il risultato con la valorizzazione delle medesime quantità al prezzo indicato nell'ultimo contratto dalle singole Aziende, ossia:

Risparmio $=\left(\mathrm{P}_{\mathrm{UslA}} * \mathrm{q}_{\mathrm{A}}+\mathrm{P}_{\mathrm{UslB}} * \mathrm{q}_{\mathrm{B}}+\ldots . . \mathrm{P}_{\mathrm{UsIN}} * \mathrm{q}_{\mathrm{N}}\right)-\left(\mathrm{P}_{\mathrm{Estav}} * \mathrm{q}_{\mathrm{A}}+\right.$ $\left.\mathrm{P}_{\text {Estav }} * \mathrm{q}_{\mathrm{B}}+\ldots . \mathrm{P}_{\text {Estav }} * \mathrm{q}_{\mathrm{N}}\right)$, dove

$\mathrm{P}_{\text {UsiN }}$ è il prezzo vecchio dell'Azienda $\mathrm{N}$

$\mathrm{q}_{\mathrm{N}}$ è la quantità indicata in delibera

$P_{\text {Estav }}$ è il prezzo spuntato dall'Estav

Con riferimento al 2005: tempo intercorrente tra indizione ed aggiudicazione del campione selezionato per la valutazione del risparmio

Con riferimento al 2006 e 2007: tempo intercorrente tra indizione o data di richiesta ed aggiudicazione per tutti gli atti contenenti tali informazioni

Lettura degli atti aventi impatto economico di tutti e tre gli Estav (anno 2007) e classificazione atti in ragione del numero di fornitori coinvolti

\section{I risultati della ricerca}

\section{Le dimensioni dell'attività degli Estav}

Nel 2007 gli importi aggiudicati dai tre Estav (Tab. 2) sono stati molto differenti: mentre Estav Centro presenta un volume quasi triplo rispetto agli altri due Enti, particolarmente contenuto appare quello di Estav Nord Ovest, considerando il numero e le dimensioni delle Aziende afferenti. Estav Centro, inoltre, a differenza degli altri due Enti, presenta un trend nell'aggiudicato in netta crescita dal 2005 al 2007.

$\mathrm{Al}$ contrario, il numero di atti aventi impatto economico risulta essere particolarmente elevato nel caso di Estav Sud Est (quadruplo rispetto ad Estav Centro e doppio rispetto a Nord Ovest): tale discrepanza è un dato consolidato sui tre anni oggetto di analisi (2007-2005). Il numero di atti è inoltre stabile negli anni, almeno per quanto riguarda Estav Centro e Sud Est, mentre Estav Nord Ovest presenta una maggiore crescita (+ 36\% dal 2005 al 2007). 
Per quanto riguarda invece il personale, l'Ente con il maggior numero di risorse umane è Estav Centro che, analogamente ad Estav Sud Est, presenta una dinamica chiaramente crescente negli anni. Estav Nord Ovest, che nel 2005 si presentava già con un'elevata dotazione di personale, ha invece mantenuto tale situazione stabile negli anni. 
Tab. 2 - Le dimensioni dell'attività degli ESTAV

\begin{tabular}{|c|c|c|c|c|}
\hline $\begin{array}{l}\text { Attività contrattuale } \\
\text { aggiudicata }\end{array}$ & Estav Nord Ovest & Estav Centro & Estav Sud Est & Totale Toscana \\
\hline Nel 2007 & 290.647 .434 & 949.359 .533 & 243.069 .487 & 1.483 .076 .454 \\
\hline Nel 2006 & 292.277.230 & 491.362 .145 & 301.705 .586 & 1.275 .001 .520 \\
\hline Nel 2005 & 135.824 .822 & 174.336 .159 & 340.459 .070 & 650.620 .051 \\
\hline \multicolumn{5}{|c|}{ L'attività contrattuale: il numero di atti aventi impatto economico } \\
\hline Numero atti & Estav Nord Ovest & Estav Centro & Estav Sud Est & Totale Toscana \\
\hline Nel 2007 & 543 & 368 & 1.295 & 2.206 \\
\hline Nel 2006 & 401 & 314 & 1.076 & 1.791 \\
\hline Nel 2005 & 399 & 393 & 1.167 & 2.380 \\
\hline \multicolumn{5}{|l|}{ Il personale } \\
\hline Numero risorse umane & Estav Nord Ovest & Estav Centro & Estav Sud Est & Totale Toscana \\
\hline Nel 2006 & 77 & 89 & 75 & 241 \\
\hline Nel 2005 & 72 & 56 & 45 & 173 \\
\hline
\end{tabular}

Tab. 3 - La tipologia degli atti

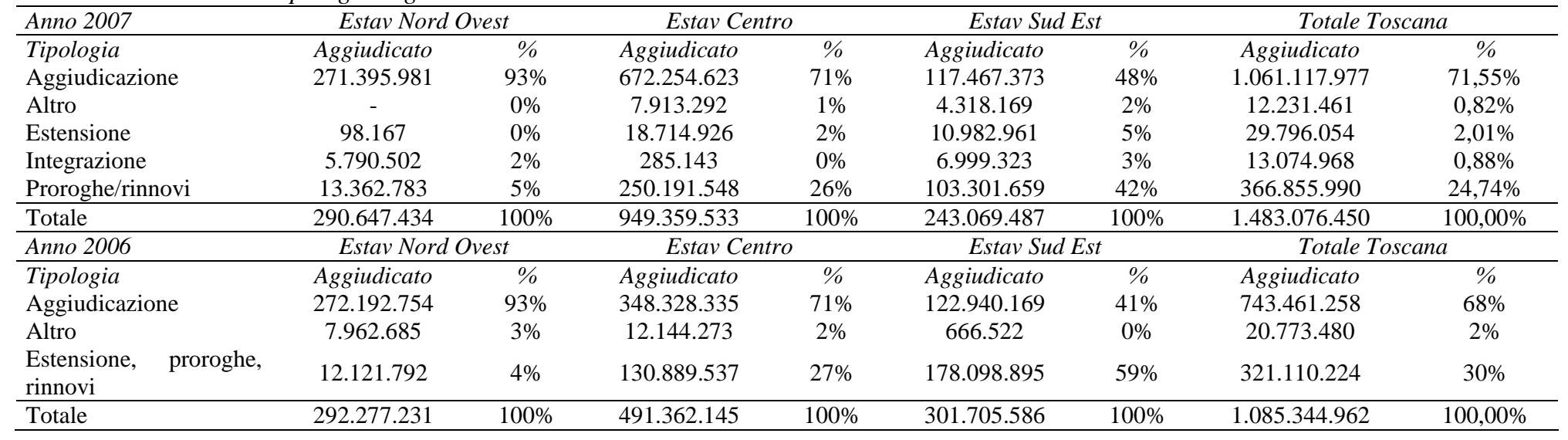




\section{L'efficienza nello svolgimento delle attività}

\section{Le tipologie di atti}

Attraverso l'individuazione delle tipologie di atti utilizzate dagli Estav (Tab. 3 e Fig. 1), è possibile comprendere quanta parte dei volumi aggiudicati derivano da nuove gare, rappresentate dalle aggiudicazioni, e quanto invece è dovuto alle proroghe e rinnovi di gare preesistenti (l'analisi per il 2006 include in un'unica classe anche le estensioni)

Si può notare come, tranne che per Estav Nord Ovest, la presenza di proroghe e rinnovi sia stata rilevante: circa il 27\% per Estav Centro (stabile tra 2006 e 2007) e ben il $42 \%$ nel caso di Estav Sud Est, per altro in diminuzione (-10\% circa) rispetto al 2006. Appare quindi evidente che larga parte dell'attività contrattuale degli Estav fa ancora riferimento alle negoziazioni precedenti, con possibile pregiudizio all'efficacia della loro azione anche se è evidente la tendenza al miglioramento.

Fig. 1 - La tipologia di atti

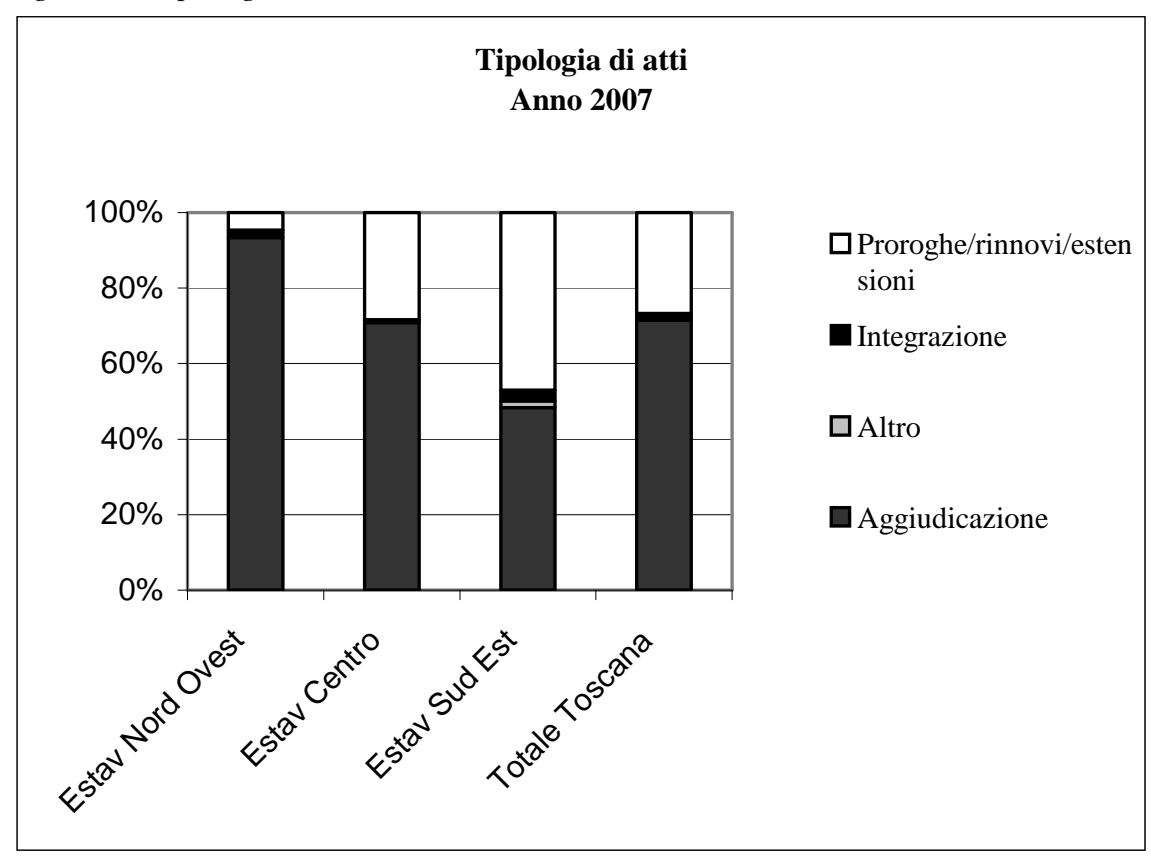


Tab. 4 - Le classi dimensionali degli atti

\begin{tabular}{|c|c|c|c|c|c|c|c|c|}
\hline \multicolumn{9}{|c|}{ L'efficienza: le classi dimensionali degli atti } \\
\hline Anпо 2007 & \multicolumn{2}{|c|}{ Estav Nord Ovest } & \multicolumn{2}{|c|}{ Estav Centro } & \multicolumn{2}{|c|}{ Estav Sud Est } & \multicolumn{2}{|c|}{ Totale Toscana } \\
\hline Classi di importo & Numero & $\%$ & Numero & $\%$ & Numero & $\%$ & Numero & $\%$ \\
\hline $0-1.000$ & 2 & $0 \%$ & 13 & $4 \%$ & 54 & $4 \%$ & $\begin{array}{c}\text { Numero } \\
69\end{array}$ & $3 \%$ \\
\hline $1.000-20.000$ & 99 & $18 \%$ & 25 & $7 \%$ & 515 & $40 \%$ & 639 & $29 \%$ \\
\hline$>20.000$, di cui: & 442 & $81 \%$ & 330 & $90 \%$ & 726 & $56 \%$ & 1.498 & $68 \%$ \\
\hline $20.000-250.000$ & $33161 \%$ & & $194 \quad 53$ & & $606 \quad 47 \%$ & & $51,27 \%$ & \\
\hline$>250.000$ & 111 & & 136 & & 120 & & $16,64 \%$ & \\
\hline Totale & 543 & $100 \%$ & 368 & $100 \%$ & 1.295 & $100 \%$ & 2.206 & $100 \%$ \\
\hline Anno 2006 & \multicolumn{2}{|c|}{ Estav Nord Ovest } & \multicolumn{2}{|c|}{ Estav Centro } & \multicolumn{2}{|c|}{ Estav Sud Est } & \multicolumn{2}{|c|}{ Totale Toscana } \\
\hline Classi di importo & Numero & $\%$ & Numero & $\%$ & Numero & $\%$ & Numero & $\%$ \\
\hline $0-1.000$ & 4 & $1 \%$ & 10 & $3 \%$ & 40 & $4 \%$ & 54 & $3 \%$ \\
\hline $1.000-20.000$ & 88 & $22 \%$ & 25 & $8 \%$ & 392 & $36 \%$ & 505 & $28 \%$ \\
\hline$>20.000$ & 309 & $77 \%$ & 279 & $89 \%$ & 644 & $60 \%$ & 1.232 & $69 \%$ \\
\hline Totale & 401 & $100 \%$ & 314 & $100 \%$ & 1.076 & $100 \%$ & 1.791 & $100 \%$ \\
\hline L'efficienza: la dimensi & media de & & & & & & & \\
\hline Dimensione media atti & Estav & d Ovest & & Centro & & ud Est & Tos & \\
\hline Nel 2007 & & 262 & & 9.781 & & 7.698 & 672.29 & \\
\hline Nel 2006 & & .871 & & 4.848 & & .396 & $€ 60$ & \\
\hline
\end{tabular}




\section{Le classi dimensionali degli atti}

Poiché è stato evidenziato che una delle modalità con cui gli Estav possono incrementare il risparmio è data dai maggiori volumi aggiudicati rispetto agli acquisti effettuati dalle singole Aziende, quest'analisi consente di comprendere se ed in che misura gli Enti si sono organizzati per aggregare le esigenze delle singole Asl e "fare volume".

A fronte di questo obiettivo, in Tab. 4 emerge in primo luogo l'anomala presenza di gare di dimensione molto contenuta (0-1.000), che non giustificano l'utilizzazione dello strumento Estav. Un secondo elemento è, soprattutto nel caso di Estav Sud Est (40\% del totale, in lieve crescita rispetto al 2006), la notevole presenza di gare di importo inferiore a 20.000 euro, tali cioè, in base ai regolamenti, da poter essere aggiudicate dalle singole Aziende.

Estav Nord Ovest ed Estav Centro presentano invece una stabile e consistente prevalenza di gare di importo superiore ai 20.000 euro, che meglio sembrano rispondere alla missione affidata.

L'analisi di approfondimento condotta nel 2007 ha consentito di evidenziare quanta parte di questi acquisti fossero per importi superiori a 250.000 euro, ossia tali da rendere necessaria una gara europea.

Nel caso di Estav Sud Est la presenza di tale tipo di gare è molto ridotta: $9 \%$ circa del totale. Nel caso di Estav Centro, invece, la percentuale è decisamente elevata: $37 \%$. Estav Nord Ovest si situa poco sopra la media dei tre Estav, con circa il $20 \%$ di gare europee.

La tendenza a ricorrere a molti atti di importo unitario contenuto si riflette sulla dimensione media degli stessi: per questa ragione Estav Sud Est presenta un valore medio molto ridotto, con trend, analogamente ad Estav Nord Ovest, in diminuzione rispetto al 2007. Estav Centro presenta invece una dimensione media ed un incremento notevoli.

Questi dati lasciano intravedere la possibilità di ampi spazi di miglioramento per i tre Estav.

\section{L'efficienza nell'impiego del personale}

Tab. 5 - La produttività del personale

\begin{tabular}{lcccc}
\hline La produttività del personale: aggiudicato/personale \\
\hline Aggiudicato/personale & $\begin{array}{c}\text { Estav Nord } \\
\text { Ovest }\end{array}$ & Estav Centro & Estav Sud Est & Toscana \\
\hline 2006 & 3.795 .808 & 5.520 .923 & 4.022 .741 & 5.290 .462 \\
2005 & 1.886 .456 & 3.113 .146 & 7.565 .757 & 3.760 .810 \\
\hline La produttività del personale: & numero atti/personale & & \\
\hline Aggiudicato/personale & Estav Nord & Estav Centro & Estav Sud Est & Toscana \\
\hline \multicolumn{7}{l}{ Ovest } & & & \\
\hline 2006 & 5,21 & 3,52 & 14,35 & 7,43 \\
2005 & 5,54 & 7,04 & 25,93 & 13,76 \\
\hline
\end{tabular}


Per avere una lettura più completa della produttività del personale, è stata calcolata sia con riferimento ai volumi aggiudicati, sia al numero di atti.

Il primo dato deriva dalla dinamica congiunta del personale e dei volumi aggiudicati, per cui si può ritenere che la maggiore consistenza del rapporto aggiudicato/personale sia indice di maggiore produttività, sia a livello di trend, sia a livello di benchmark. Il secondo dato, ossia la produttività intesa come rapporto tra il numero di atti ed il personale, derivando, oltre che dalle variazioni di personale, anche da quelle connesse al numero di atti, è di più dubbia interpretazione. In effetti, si potrebbe ritenere che, anche alla luce della precedente analisi circa la composizione degli atti in termini dimensionali e tipologici, un elevato rapporto tra numero atti e personale non sia indice di particolare efficienza, ma di tendenza alla frammentazione dell'attività.

In questo senso appare particolarmente positiva la situazione di Estav Centro che nel 2006 risulta essere il più efficiente rispetto al volume aggiudicato, con trend molto positivo rispetto al 2005, ed una forte riduzione degli atti aggiudicati. Estav Nord Ovest presenta una situazione più critica, ma in miglioramento rispetto al 2005; Estav Sud Est presenta una buona efficienza rispetto al volume nel 2006, ma in netto peggioramento rispetto al 2005 e con un rapporto numero atti/personale decisamente troppo elevato rispetto alla media.

\section{L'efficacia dell'azione degli Estav}

\section{Il risparmio effettivamente conseguito}

Come illustrato nella sezione sulla metodologia, relativamente alla valutazione del risparmio sono state adottate due diverse modalità di individuazione del campione.

Tab. 6 - Il risparmio conseguito-valutazione delle delibere aggiudicate nell'anno 2007

\begin{tabular}{|c|c|c|c|}
\hline & $\begin{array}{l}\text { Importo aggiudicato } \\
\text { del campione }\end{array}$ & $\begin{array}{l}\text { Importo economie } \\
\text { riscontrate nel } \\
\text { campione }\end{array}$ & $\begin{array}{c}\text { Economie riscontrate nel } \\
\text { campione/importo } \\
\text { aggiudicato del } \\
\text { campione }\end{array}$ \\
\hline Totale Regione & $566.717 .577,24$ & $33.527 .024,66$ & $5,9 \%$ \\
\hline
\end{tabular}


Tab. 7 - Il risparmio conseguito-valutazione delle delibere aggiudicate nell'anno 2005

\begin{tabular}{lccc}
\hline & Estav Nord Ovest & Estav Centro & Estav Sud Est \\
\hline Risparmio effettivo & n.v. & 3.745 .358 & 11.745 .837 \\
$\begin{array}{l}\text { Risparmio/Importo } \\
\text { aggiudicato }\end{array}$ & n.v. & $2,40 \%$ & $3,45 \%$ \\
\hline
\end{tabular}

Nel 2007 (Tab. 6) il risparmio conseguito a livello regionale risulta essere particolarmente consistente e pari a circa il $6 \%$ dell'aggiudicato. Il dato appare molto significativo considerando che è calcolato senza considerare il fenomeno inflativo ed il processo generalizzato di aumento dei prezzi di acquisto dei beni e servizi nella Pubblica Amministrazione intorno al 3\%.

L'analisi relativa ai dati del 2005 (Tab. 7), con il limite della non valutabilità riscontrata nel caso di Estav Nord Ovest, evidenzia il contributo apportato dai singoli Estav, particolarmente elevato per Sud Est e, confrontando tali dati con quello relativo al 2007, lascia ipotizzare un trend di crescita del risparmio.

\section{La durata delle procedure}

Tab. 8 - La durata delle procedure

\begin{tabular}{ccccc}
\hline $\begin{array}{c}\text { Tempo intercorrente } \\
\text { tra indizione ed } \\
\text { aggiudicazione }\end{array}$ & $\begin{array}{c}\text { Estav Nord } \\
\text { Ovest }\end{array}$ & Estav Centro & Estav Sud Est & Media Toscana \\
\hline 2007, di cui: & 154 & 159 & 106 & 140 \\
$\quad<250.000$ euro & 137 & 129 & 93 & 119 \\
$\quad>250.000$ euro & 178 & 195 & 230 & 201 \\
2006 & 125 & 136 & 104 & 122 \\
2005 (parziale) & 246 giorni & $\begin{array}{c}\text { Rilevabile solo } \\
\text { per alcune gare: }\end{array}$ & 239,8 giorni & 242 \\
& $(8$ mesi circa) & \\
& \multicolumn{3}{c}{ (8 mesi } \\
\hline
\end{tabular}

La durata delle gare indica quanto l'Estav è in grado di reagire prontamente al verificarsi di richieste da parte delle Aziende. E' da notare che in questa presentazione è incluso solo il tempo intercorrente tra indizione ed aggiudicazione, ed è quindi al netto del periodo precedente, ossia dalla richiesta effettuata dall' Azienda, alla raccolta da parte dell'Estav di eventuali altre adesioni all'acquisto, fino all'indizione vera e propria della gara.

Si può notare che, con la sola eccezione di Estav Sud Est, i tempi sono in via di incremento tra 2006 e 2007. Particolarmente veloce in ambedue gli anni risulta essere in media Estav Sud Est. L'analisi di dettaglio del 2007, che evidenzia le gare di importo superiore ai 250.000 euro (per le quali è prevista l'indizione di gare europee, con tempi tecnici prolungati), aiuta a leggere meglio la situazione dei tre Estav. 
Estav Sud Est è probabilmente mediamente più veloce in virtù del fatto che effettua in maniera rilevante aggiudicazioni di importo contenuto.

Gli altri due Estav, ed in particolare Nord Ovest, agevolato forse in questo dall'utilizzazione delle gare online, si rivelano invece più rapidi (di un mese circa) rispetto ad Estav Sud Est nella gestione delle gare di dimensione europea.

\section{Il numero di fornitori invitati alla gara}

Tab. 9 - Il numero fornitori coinvolti nelle singole gare

\begin{tabular}{lcccccccc}
\hline $\begin{array}{l}\text { Anno 2007 } \\
\text { Solo Aggiudicazioni }\end{array}$ & Estav Nord Ovest & \multicolumn{2}{c}{ Estav Centro } & Estav Sud Est & \multicolumn{2}{c}{ Totale Toscana } \\
\hline Classi di fornitori & Numero & $\%$ & Numero & $\%$ & Numero & $\%$ & Numero & $\%$ \\
1 & 162 & $36 \%$ & 62 & $29 \%$ & 231 & $41 \%$ & 455 & $37 \%$ \\
$2-5$ & 147 & $33 \%$ & 77 & $36 \%$ & 263 & $47 \%$ & 487 & $40 \%$ \\
$6-10$ & 62 & $14 \%$ & 56 & $26 \%$ & 62 & $11 \%$ & 180 & $15 \%$ \\
$>11$ & 77 & $17 \%$ & 20 & $9 \%$ & 9 & $2 \%$ & 106 & $9 \%$ \\
\hline Totale & 448 & $100 \%$ & 215 & $100 \%$ & 565 & $100 \%$ & 1228 & $100 \%$ \\
\hline
\end{tabular}

L'analisi della letteratura ha evidenziato che una delle metodologie con cui l'Estav può generare risparmio di spesa sia data dal conseguimento di un maggiore potere di mercato. Una delle modalità con cui accrescere il potere all'interno del mercato, oltre che attraverso l'aggregazione delle gare, è anche garantirne la massima apertura ed il coinvolgimento del massimo numero di fornitori. Dall'analisi emerge chiaramente che, invece, soprattutto nel caso di Estav Sud Est, vi è una notevole quantità di gare per cui viene invitato un unico fornitore, aspetto che fa ritenere che l'obiettivo di accrescimento del potere degli Estav rispetto ai fornitori sia lungi dall'essere conseguito, ma anche che vi siano ampi spazi per il miglioramento dell'efficacia della loro azione.

\section{Conclusioni}

Rispetto alle indicazioni fornite dalla letteratura certamente l'esperienza degli Estav toscani permette di evidenziare che anche nella sanità pubblica, in un contesto organizzativo a rete, basato su rapporti di tipo collaborativo e non competitivo, i vantaggi complessivi ottenuti risultano di notevole entità. In un ambito infatti di risorse limitate rispetto all'evoluzione e all'incremento dei bisogni della popolazione, la possibilità di ridurre il costo degli acquisti di beni e servizi, che risultano la componente di spesa più in crescita nel contesto sanitario, di $5-6$ punti percentuali appare un risultato eccezionale. Certamente influisce non solo la bontà della nuova proposta di assetto organizzativo di sistema ma probabilmente anche la debolezza della soluzione organizzativa precedente, in cui le funzioni erano strutturate presso le aziende e non erano sottoposte ad un processo rigoroso di governo e di controllo dei risultati. 
Il risultato appare ancora più interessante e la soluzione organizzativa valida alla luce del fatto che gli Estav non hanno avuto la libertà sia di scegliersi le risorse umane operanti nelle aziende sia di assumere profili specifici dall'esterno, con competenze ed esperienze nell'ambito della gestione acquisti e logistica. Questi margini di manovra, limitati anche dalla presenza di inevitabili resistenze da parte delle Aziende, costrette a cedere ambiti di attività e potere, hanno determinato notevoli difficoltà e rallentamenti nel trasferimento delle funzioni agli Estav, ma non hanno ridotto gli innegabili vantaggi conseguiti dal sistema.

In particolare il risultato di cost saving ottenuto rappresenta certamente uno dei fattori alla base della capacità del sistema sanitario toscano nel suo complesso di mantenere le condizioni di equilibrio economico finanziario, senza ricorrere ad imposte aggiuntive locali o a ticket.

La differente performance registrata nei tre Estav comunque evidenzia che il sistema ha ancora spazi di notevole miglioramento e il confronto sistematico dei risultati può permettere sia la valorizzazione delle best practices sia l'attivazione di processi efficaci di apprendimento organizzativo.

In questo senso appare importante creare le condizioni sistematiche per il confronto, attraverso:

- l'omogeneizzazione delle modalità di reportistica dei risultati e delle attività dei tre Estav;

- l'attivazione di flussi informativi omogenei per la raccolta delle informazioni rilevanti;

- un confronto sistematico dei risultati ottenuti sia tra Estav e le Aziende sanitarie per Area Vasta, sia a livello regionale;

- l'introduzione di un sistema di incentivazione che rafforzi il ruolo complementare nel sistema dei diversi attori (Estav e Aziende) e favorisca il gioco di squadra di tutte le componenti.

Queste attenzioni possono evitare ciò che in letteratura è stato spesso rilevato, ossia di porre grande attenzione alla fase di avviamento dei processi di esternalizzazione senza costruire un sistema di monitoraggio e di valutazione dei risultati che permetta di garantire il loro consolidamento nel tempo, mediante anche eventuali correttivi all'azione.

Rispetto agli altri obiettivi (la razionalizzazione del portafoglio dei fornitori e la conseguente maggiore efficienza nelle relazioni di approvvigionamento; la possibilità di attrarre, grazie ai maggiori volumi acquistati, imprese precedentemente non interessate a rifornire le singole Aziende; la standardizzazione dei beni e dei servizi richiesti dalle Aziende, utile per procedere all'aggregazione della domanda) che l'introduzione degli Estav si prefiggeva, gli elementi a disposizione sono ancora insufficienti per procedere ad una valutazione esaustiva.

Certamente i risultati ottenuti sono ancora migliorabili e forse meno soddisfacenti rispetto alle aspettative. Questo è dovuto alla lentezza con cui le organizzazioni pubbliche cambiano e alla difficoltà di attivare comportamenti differenti soprattutto relativamente all'ultimo aspetto ricordato, che richiede un notevole coinvolgimento delle Aziende e dei professionisti. 
Rispetto infine alla possibilità di poter usufruire a livello di sistema di una maggiore flessibilità organizzativa (Gandolfi, 2000), in quanto la sostituzione della struttura gerarchica interna con una esterna, regolata tramite contratto, consente un rapido adeguamento alle variazioni di carico di lavoro (Valentini, 1999), modificando anche la struttura dei costi aziendali, di cui viene accresciuta la componente variabile rispetto a quella fissa (Gregori, 2001) l'esperienza toscana non ha portato evidenze confermative a causa della rigidità contrattuale presente nel rapporto di lavoro. L'unica flessibilità riscontrata è stata la mobilità interna tra Aziende ed Estav, tra l'altro complessa e difficile, realizzata solo mediante interventi di incentivazione specifici.

Malgrado quindi queste rigidità tipiche del settore pubblico, i risultati conseguiti con l'introduzione degli Estav nel sistema sanitario pubblico toscano appaiono complessivamente positivi e indicativi di un percorso da intraprendere. Per massimizzare ulteriormente il risultato conseguibile, sarebbe comunque auspicabile per i sistemi sanitari pubblici interessati a seguire questa strada una maggiore incisività e rapidità.

\section{Bibliografia}

Anderson J.C., Narus J.A. (2004). Business Market Management. New Jersey: Prentice Hall.

Brusoni M., Marsilio M. (2007). La gestione centralizzata degli approvvigionamenti nei sistemi sanitari regionali. In: Annessi Pessina E., Cantù E., a cura di, Rapporto OASI 2007. L'aziendalizzazione della sanità in Italia. Milano: EGEA.

Brusoni M., Cappellaro G., Marsilio M. (2008). Processi di accentramento degli approvvigionamenti in sanità: una prima analisi d'impatto. In: Annessi Pessina E., Cantù E., a cura di, Rapporto OASI 2007. L'aziendalizzazione della sanità in Italia. Milano: EGEA.

Coase R.H. (1937). The Nature of the Firm. Economica, 4: 386-405.

Cozzi G., Ferrero G. (2004). Marketing. Principi, metodi, tendenze evolutive. Torino: Giappichelli.

Cravedi, B. (2003). L'esperienza toscana: strategia e programma di azione dei Consorzi di Area Vasta. In Miolo Vitali P., Nuti S., a cura di, Ospedale in rete e reti di ospedali: modelli ed esperienze a confronto. Milano: Franco Angeli.

Del Vecchio M. (2005). Il sistema di approvvigionamenti e distribuzione dei beni in sanità. Alcune considerazioni di sintesi. In Nuti S., a cura di, Innovare in sanità. Esperienze internazionali e sistema toscano a confronto. Pisa: Edizioni ETS.

Del Vecchio M., Rossi A.G. (2004). I Consorzi di Area Vasta in Toscana: il caso del CAVC tra innovazione gestionale e trasformazione istituzionale. In: Annessi Pessina E., Cantù E., a cura di, Rapporto OASI 2004. L'aziendalizzazione della sanità in Italia. Milano: EGEA.

Dominijanni M., Nante N. (2007). ESTAV: un modello di efficienza in sanità, XII Conferenza Annuale AIES, Firenze, 18-19 ottobre 2007. 
Gadde L.-E., Jonsson P. (2007). Outsourcing and Supply Network Performance consequences of sourcing and producing in low-cost countries, Proceedings of the 23rd IMP-Conference, Manchester, August 29-31.

Gadde L.-E., Hakansson H. (1994). The Changing Role of Purchasing: Reconsidering Three Strategic Issues. European Journal of Purchasing and Supply Management, $1,1: 27-35$.

Gandolfi V. (2000). Economia e Gestione delle Imprese, Parma: Editrice Campus.

Gregori G. (2001). L'evoluzione del processo di outsourcing nelle strategie di sviluppo delle imprese, Torino: Giappichelli Editore.

Hakansson H., Gadde L.E. (2002). Supply Network Strategies. London: John Wiley \& Sons.

Hines P. (1996). Purchasing for lean production: the new strategic agenda, International Journal of Purchasing and Materials Management, 32, 1: 2-10.

Kakabadse A., Kakabadse N. (2001). Outsourcing in the Public Services: a Comparative Analysis of Practice, Capability and Impact, Public Administration Development, 21: 401-413.

Kralijc P. (1983). Purchasing Must Become Supply Management, Harvard Business Review, September-October: 109-117.

Macinati M S. (2006). L'outsourcing nel SSN: motivazioni, caratteristiche e risultati. In: Annessi Pessina E., Cantù E., a cura di, Rapporto OASI 2006. L'aziendalizzazione della sanità in Italia. Milano: EGEA.

Meneguzzo M., Roncetti L., Rossi A. G. (2004). Centrali di acquisto, servizi in rete ed "Aree Vaste". Una prima valutazione delle esperienze in atto, Mecosan, 52: 115-133.

Miolo Vitali P., Nuti S., a cura di (2003). Ospedale in rete e reti di ospedali: modelli ed esperienze a confronto. Milano: Franco Angeli.

Momme J., Hvolby H.H. (2002). An Outsourcing Framework: Action Research in the Heavy Industry Sector, European Journal of Purchasing \& Supply Management, 8: 185-196.

Nuti S., a cura di (2005). Innovare in sanità. Esperienze internazionali e sistema toscano a confronto. Pisa: Edizioni ETS.

Nuti S., a cura di (2008). La valutazione della performance in sanità. Bologna: Il Mulino.

Padovani E. (2004). Il governo dei servizi pubblici locali in outsourcing: il controllo dell'efficacia. Milano: Franco Angeli.

Powell W.W. (1987). Hybrid organizational arrangements: new forms or transitional development?, California Management Review, Fall: 67-87.

Prahalad C.K., Hamel G. (1990). The Core Competence of the Corporation, Harvard Business Review, May-June: 79-91.

Quinn J.B. (2000). Outsourcing Innovation: the New Engine of Growth, Sloan Management Review, 41, 4: 13-28.

Quinn J. B., Hilmer F. G. (1994). Strategic outsourcing, Sloan Management Review Summer: 43-55.

Richardson, G.B. (1972). The organization of industry, The Economic Journal, 82, 327: 883-896. 
Scarsi R. (2004). Gli operatori logistici: caratteri strutturali e mercato, Torino: Giappichelli Editore.

Schuller M., Hubner U. (2008). Logistics Service and Beyond. In: Hubner U., Elmhorst M.E., eds., E-business in Healthcare. From Procurement to Supply Chain Management, London: Springer-Verlag.

Spekman R.E., Kamauff J.W., Salmond D.J. (1994). At Last Purchasing is becoming strategic, Long Range Planning, 27, 2: 76-84.

Tayles M., Drury C. (2000). Moving from Make/Buy to Strategic Sourcing: The Outsource Decision Process, Long Range Planning, 34: 605-622.

Tunisini A. (2003). Supply chains e strategie di posizionamento, Roma: Carocci Editore.

Turchetti G. (2005). La politica degli acquisti di beni e servizi in ambito pubblico: le tendenze in atto nel settore sanitario, Milano: FrancoAngeli Editore.

Valentini S. (1999). Gestire l'outsourcing: i passi fondamentali per avere successo in un processo di ristrutturazione, Milano: FrancoAngeli Editore.

Venkatesan R. (1992). Strategic sourcing: to make or not to make, Harvard Business Review, November/December: 98-107.

Williamson O.E (1975). Market and Hierarchies. Analysis and Antitrust Implications. A Study in the Economics of Internal Organizations, New York: The Free Press.

Legge Regionale Toscana 22/2000.

Delibera Consiglio Regione Toscana 144/2000.

Delibera Giunta Regione Toscana 1021/2005.

Delibera Giunta Regione Toscana 617/2006.

Delibera Giunta Regione Toscana 317/2007. 